\title{
BMJ Open Prevalence of maternal psychological disorders after immediate postpartum haemorrhage: a repeated cross-sectional study - the PSYCHE* study protoco
}

\author{
Marine Pranal, ${ }^{1,2}$ Anne Legrand, ${ }^{1,2}$ Ingrid de Chazeron, ${ }^{3,4}$ Pierre-Michel Llorca, ${ }^{3,4}$ \\ Françoise Vendittelli ${ }^{1,2,5}$
}

To cite: Pranal M, Legrand A, de Chazeron I, et al. Prevalence of maternal psychological disorders after immediate postpartum haemorrhage: a repeated cross-sectional study - the PSYCHE* study protoco. BMJ Open 2019;9:e027390. doi:10.1136/ bmjopen-2018-027390

- Prepublication history for this paper is available online. To view these files, please visit the journal online (http://dx.doi. org/10.1136/bmjopen-2018027390).

Received 07 November 2018 Revised 03 May 2019 Accepted 08 July 2019

\section{Check for updates}

(c) Author(s) (or their employer(s)) 2019. Re-use permitted under CC BY-NC. No commercial re-use. See rights and permissions. Published by BMJ.

For numbered affiliations see end of article.

\section{Correspondence to} Marine Pranal;

m_julien@chu-

clermontferrand.fr

\section{ABSTRACT}

Introduction The main objective of this study is to assess the prevalence of depression at 2, 6 and 12 months postpartum in women who have had an immediate postpartum haemorrhage (PPH) (blood loss $\geq 500 \mathrm{~mL}$ within 24 hours of delivery). The secondary objectives are to assess the prevalence of anxiety and post-traumatic stress disorder among these women and to evaluate the prevalence of psychological disorders according to the severity of the PPH.

Methods and analysis This repeated, cross-sectional, single-centre study will take place at the Clermont-Ferrand University Hospital (France). The population will comprise a cohort of women giving birth at a term $\geq 22$ weeks of gestation.

For each woman with a PPH (exposed), two women without PPH (unexposed) will be included: the women who give birth immediately before and immediately after her. The PPH will be managed according to French guidelines. The principal endpoint is the prevalence of depression, measured by the Edinburgh Postnatal Depression Scale (EPDS). The intervention will consist of four surveys including various self-completed questionnaires: the first during the immediate postpartum (Post-Delivery Perceived Stress Inventory (PDPSI), Spielberger'sState-Trait Anxiety Inventory (STAI)-Y-A and Y-B and Mini-International Neuropsychiatric Interview (M.I.N.I.) 5.0.0), then at 2 months (EPDS, STAI-Y-A, Generalised Anxiety Disorder (GAD-7) and Revised Impact of Event Scale (IES-R)), and finally at 6 months and 1 year postpartum (EPDS, STAI-Y-A, GAD-7, M.I.N.I. 5.0.0 and IES-R). The study will include 1542 women -514 with $\mathrm{PPH}$.

Ethics and dissemination The institutional review board (IRB) approved the study on 14 February 2017 (IRB Sud Est VI: NAU1243).

Results will be reported in peer-reviewed journals and at scientific meetings. Findings from the study will be useful for individualising medical follow-up after childbirth, especially for woman who experienced a PPH, but also more generally in increasing birth professionals' awareness of effects of trauma. The evidence obtained might also lead to modifying practices and including this recommendation in French guidelines on PPH.

Trial registration number NCT03120208.

\section{Strengths and limitations of this study}

- The study will include a large number of women who will be followed up for 1 year (514 exposed women with postpartum haemorrhage (PPH) and 1028 non-exposed with no PPH).

- Women from both groups will complete each questionnaire at the same times relative to delivery (at inclusion, and 2, 6 and 12 months afterwards).

- The main limitation of this single-centre study based on self-administered questionnaires may be a high non-response rate, especially among the unexposed women; for this reason, we are including two unexposed women for each exposed woman.

\section{INTRODUCTION}

\section{Background and rationale}

During the postnatal period, women may develop psychological disorders that can range in severity from 'baby blues' (15\% to $80 \%$ of women) to postpartum depression to puerperal psychosis. ${ }^{1-18}$ The prevalence of postpartum depression in the general population has been estimated to vary from $10 \%$ to $20 \% \cdot{ }^{9-16}$ In France, studies have reported a prevalence of $17 \% .^{19}{ }^{20}$ Studies that have measured postpartum post-traumaticstress disorder (PTSD) observed a mean prevalence of $4.0 \%$ (95\% CI 2.77 to 5.71 ) in community samples and a prevalence of $18.5 \%$ (95\% CI 10.6 to 30.38) after birth for women in highrisk groups. ${ }^{21-26}$ The estimates of prevalence of anxiety during this period range from $8.7 \%$ to $40.4 \% .^{27-29}$

Studies have shown that postpartum risks of onset of depression, post-traumatic stress and anxiety are higher among women whose pregnancy included obstetrical complications, compared with women with uneventful pregnancies. $^{21-24} \quad 30$ Other studies have shown that caesarean and operative vaginal 
deliveries increase the risk of depression and post-traumatic stress. ${ }^{12} 232531$

A history of psychiatric disease or psychological disorders before the pregnancy is also a risk factor for both postpartum depression and post-traumatic stress disorder. ${ }^{32} 33$ Inversely, for women with normal deliveries, the risk of post-traumatic stress is lower when the woman feels that she was supported during the delivery by the healthcare team and her partner and that she was able to control the management of her labour. ${ }^{22}$

Immediate postpartum haemorrhage (PPH) concerns around $3.4 \%$ of vaginal deliveries $(\geq 500 \mathrm{~mL})$ and $2.8 \%$ of caesareans $(\geq 1000 \mathrm{~mL})$ in France, where it is the leading cause of maternal death. ${ }^{3435}$ According to the 2014 French guidelines, $\mathrm{PPH}$ is defined by blood loss $\geq 500 \mathrm{~mL}$ in the first 24 hours after birth, regardless of mode of delivery. ${ }^{35}$ Management of severe PPH can require procedures that are medical (blood transfusion) or surgical (arterial ligation, emergency hysterectomy, etc) or involve interventional radiology. ${ }^{35}$ These potential serious consequences of PPH may lead to a higher rate of psychological disorders in these women than in those without PPH. However, current French guidelines do not raise the issue of potential psychological disorders after PPH nor do they recommend their screening and their management for women with PPH.

Few published studies have examined the psychological impact of immediate PPH. ${ }^{36-38}$ One study interviewed women by telephone, ${ }^{37}$ and two others sent women self-administered questionnaires by email at both 2 and 4 months postpartum, with individual telephone reminders if they failed to respond. ${ }^{35-38}$ These studies present methodological problems including the use of questionnaires that have not been validated in English or in French, ${ }^{36} 37$ the retrospective nature of the studies ${ }^{37}$ and the large number of women lost to follow-up, information bias (women not all interviewed at the same intervals after delivery), recall bias, small sample size, failure to adjust for antenatal risk factors known to affect postpartum depression, PTSD or anxiety. ${ }^{36-38}$ One study included only women with vascular embolisation, ${ }^{37}$ while another multicentre study considered only women who had lost $>1500 \mathrm{~mL}$ blood or had a haemoglobin concentration of $7 \mathrm{~g} / \mathrm{dL}$ or a drop in the haemoglobin concentration of $4 \mathrm{~g} / \mathrm{dL}$ or more in postpartum, ${ }^{38}$ that is, only women with severe $\mathrm{PPH}$, rather than all $\mathrm{PPH}$. The studies were also limited to a shorter postpartum period (4 months for Thompson et al) ${ }^{36-38}$ Clinicians with relevant expertise recommend that these assessments also be performed at 6 months and 1 year. $^{910}$

\section{Objectives}

The main objective of our study is to assess the prevalence of depression at 2, 6 and 12 months postpartum among women with an immediate PPH.

Secondary objectives are to assess, at the same intervals, the prevalence (and severity) of anxiety and PTSD, as well as the prevalence (and severity) of depression according to the severity of the haemorrhage (mild-moderate PPH: $500 \mathrm{~mL}$ to $<1000 \mathrm{~mL}$, compared with severe PPH, defined as $\geq 1000 \mathrm{~mL}$ ) and according to the specific types of 'second-line' medical management (transfusion, vascular embolisation, etc). The trends in the prevalence of depression over the three study periods will be analysed.

\section{METHODS AND ANALYSIS}

\section{Status of ongoing study}

This protocol is Version 9, as modified on 27 July 2016. The study began in April 2017 after it received all authorisations necessary under French law. The recruitment period should be ended before December 2019. See the trial registration data for all registration details (table 1). This PSYCHE protocol has followed published guidelines for cross-sectional studies (STROBE 2007-V4 Checklist).

\section{Study design}

This repeated, cross-sectional, descriptive and aetiological study will take place within a cohort of women giving birth, comparing each woman with a PPH (exposed) to two women without PPH (unexposed).

\section{Setting}

This is a single-centre study, performed at the University Hospital Centre in Clermont-Ferrand, France.

\section{Participants}

Women are eligible for inclusion regardless of parity and mode of delivery (vaginal or caesarean) if they give birth at a term $\geq 22$ weeks' gestation or, if the term is unknown, to a fetus with a birth weight $\geq 500 \mathrm{~g}$, and they are covered by the French health insurance fund (CNAMTS). Women will be excluded if they are younger than 18 years, do not understand French, refuse to participate or do not give birth at the Clermont-Ferrand University Hospital Centre Maternity Unit but are instead secondarily transferred postpartum. This term corresponds to the definition of viability according to both WHO and the French Clinical Practice Guidelines. We chose not to exclude the women with very preterm births so that our sample would be as representative as possible of the general population of women giving birth in French hospitals. Moreover, in light of the overall number of women in our study (1542 women will be included), these women should account for a small minority of the cases.

The exposed women will be those with an immediate PPH (defined as a blood loss $\geq 500 \mathrm{~mL}$ during the 24 hours after birth) after a vaginal or caesarean delivery. The non-exposed women will be those without an immediate $\mathrm{PPH}$.

\section{Patient and public involvement}

No patients or public members were involved in the development of the research question, the study design or recruitment or outcome measures. The questionnaires were pilot tested among women in advance. We ensured the clarity and acceptability (filling time) of the 
Table 1 Trial registration data for the PSYCHE study

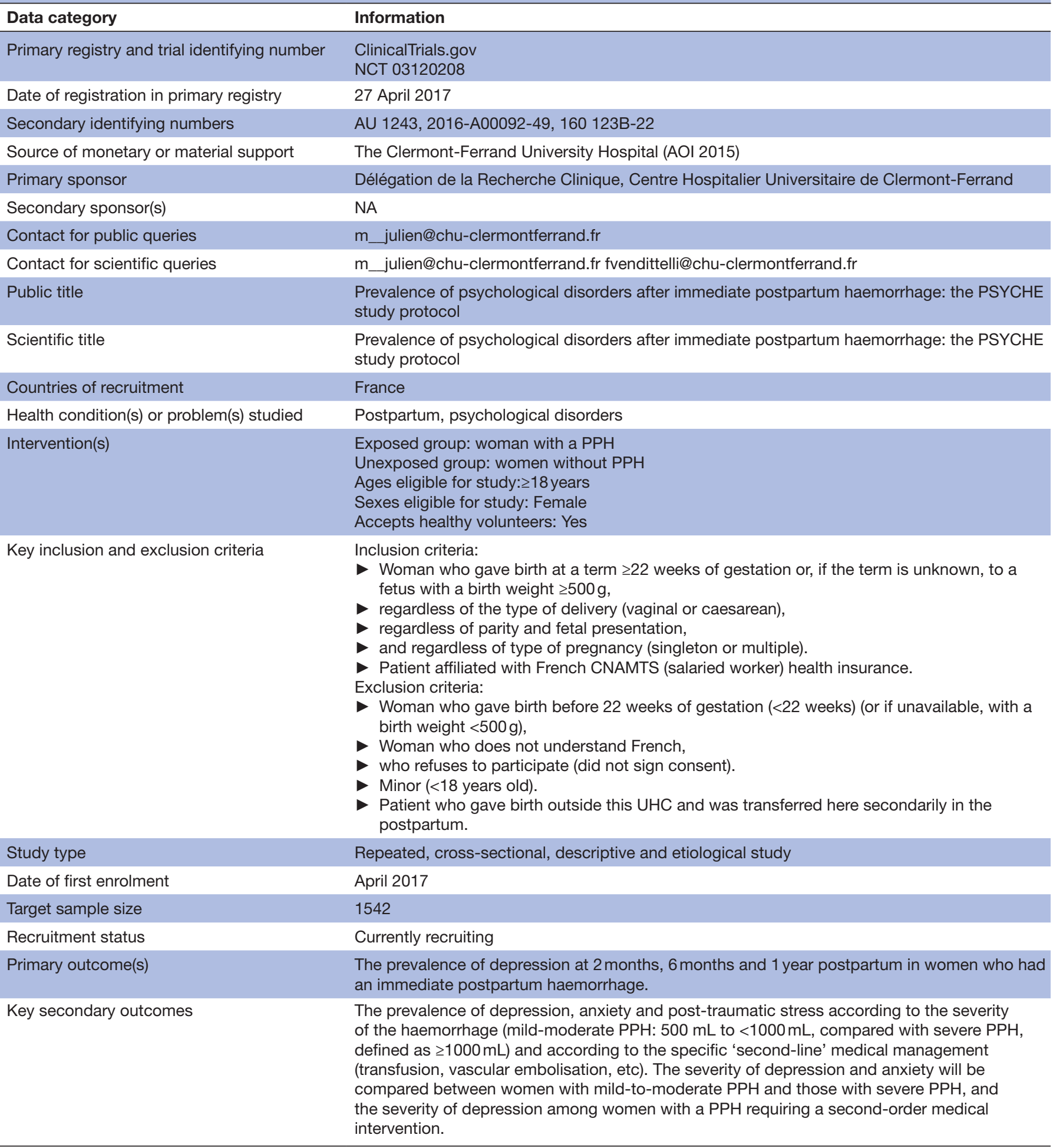

PPH, postpartum haemorrhage; UHC, University Hospital Center.

questionnaires by administering it to 10 women. This pretest showed no difficulties in understanding and no adaptations were therefore required. The results of this trial will be disseminated by publication in international peer-reviewed scientific journals and by presentations at international and domestic conferences. If the trial results warrant changes in the standard treatment of immediate postpartum haemorrhage, this trial will assist the implementation of a new standard of care in our maternity unit to improve systematic screening of women with psychological needs and ensure they receive adequate management of any psychological problems after delivery and after discharge. It might also provide evidence to influence the standard of care 
and guidelines for PPH management, in France and elsewhere.

\section{Variables}

For our primary outcome, the prevalence of depression, we will use the Edinburgh Postnatal Depression Scale (EPDS) to detect and quantify depressive symptoms in the postpartum period (with a discrimination threshold $\geq 11),{ }^{39}$ together with the Mini-International Neuropsychiatric Interview (M.I.N.I.) 5.0.0, which studies the principal Axis 1 psychiatric disorders of Diagnostic and Statistical Manual of Mental Disorders, Fourth edition (DSM-IV) (American Psychiatric Association), including depression. $^{40}$

The study will also use Spielberger's State-Trait Anxiety Inventory (STAI-Y), which enables the evaluation of anxiety as a personality trait (form Y-B, discrimination threshold $\geq 48$ ) and anxiety as an emotional state linked to a particular event (form Y-A, discrimination threshold $\geq 46)^{4142}$; the Generalised Anxiety Disorder (GAD-7 scale, with seven items and a discrimination threshold $\geq 10$ ), which enables screening for generalised anxiety and the measurement of its severity ${ }^{43-45}$ and the Revised Impact of Event Scale (IES-R) (with a discrimination threshold $\geq 30$ ), created by Weiss and Marmar $(1997)^{46}$ to assess the presence of traumatic stress linked to violent events. IES-R is able to differentiate subjects with acute stress from those with post-traumatic stress. ${ }^{48}$ The stress perceived by women at delivery in the immediate postpartum period will be assessed by the post-delivery perceived stress inventory (PDPSI). ${ }^{44} 4799$ Other relevant individual, social and medical covariables, especially useful as confounding and especially prognostic factors for the study, will be extracted from the electronic medical file. These include history of depression, anxiolytic treatment before delivery, psychological or psychiatric care before, during and after delivery, type of delivery, gestational age at birth and the child's status at birth, etc. The women's family situation will be known from their individual medical files, so that we will be able to adjust our results according to parity and the number of children already in the home.

Blood loss will be estimated quantitatively with a collector bag for vaginal deliveries and by the aspiration and weighing of blood in used supplies for caesareans, as usual in our maternity unit. The PPH will be managed according to the most recent national guidelines, issued in December 2014. ${ }^{35}$

\section{Data sources/measurement}

The study will use scales validated in the general population and in French for the measurement of psychological status (figure 1). ${ }^{39-49}$ The intervention will consist of surveys conducted by self-administered questionnaires in the immediate postpartum and at 2, 6 and 12 months postpartum (figure 1). Completion of each questionnaire takes approximately $20 \mathrm{~min}$, and the women will have only four questionnaires, distributed over a full year. The women are clearly informed of this timing at inclusion

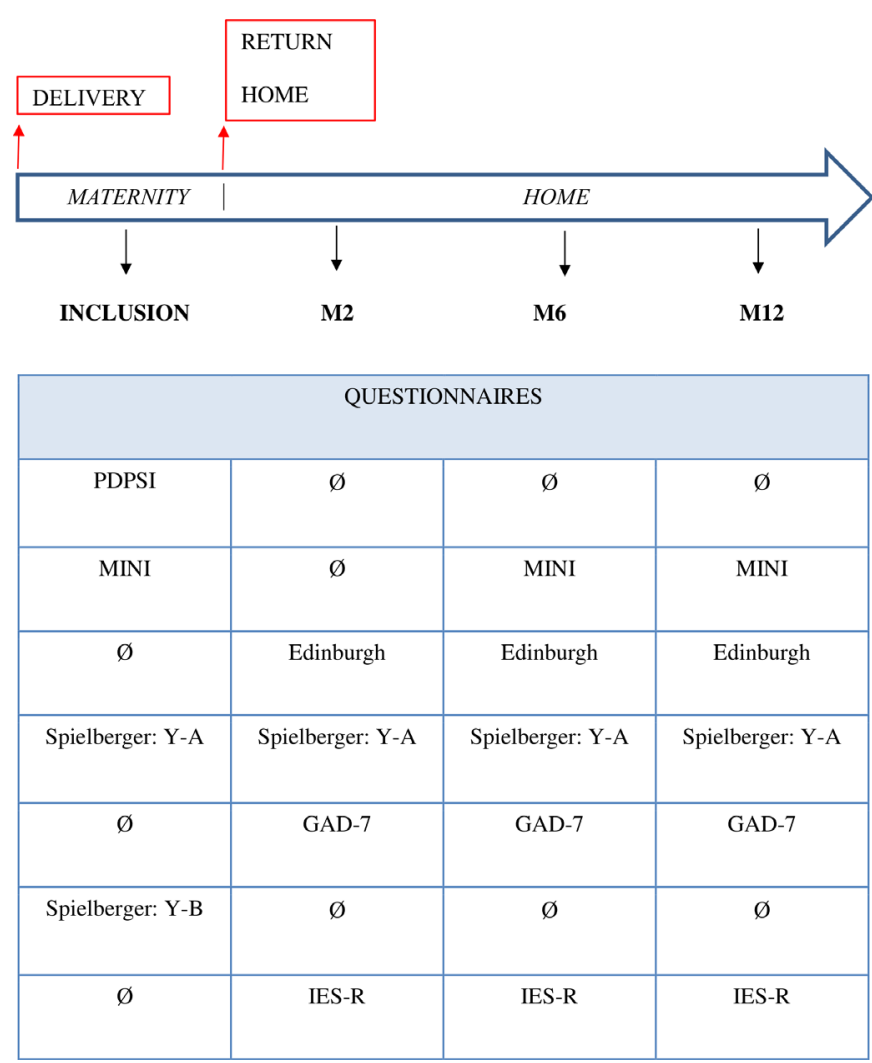

Figure 1 Schedule of enrolment, interventions and assessments of women for the PSYCHE study. GAD,Generalised Anxiety Disorder; IES-R, Revised Impactof Event Scale; M, month; MINI, MiniInternationalNeuropsychiatric Interview; PDPSI, post-delivery perceived stressinventory.

and can refuse to participate if it seems too difficult to them. These are multiple-choice questionnaires and accessible and quick to complete, since they require no writing. They can all be completed directly online and can be stopped and continued later, at a more convenient moment. We have attempted to facilitate as much as possible for the completion of the questionnaires.

For each woman with a PPH (exposed), the study will include two women without PPH (unexposed): the eligible women who gave birth immediately before and immediately after her. If one of these two unexposed women refuses to participate, another woman immediately just before or after them will be included.

Information and inclusion will take place in the immediate postpartum period at the Clermont-Ferrand University Hospital Centre, France. An investigator (midwife or obstetrician) will inform eligible women about the study. This investigator will provide them with a written information sheet and obtain their written consent before their discharge. The women of the cohort will complete each questionnaire at the same interval after their date of delivery (immediate postpartum, and 2, 6 and 12 months afterwards). They will receive an email asking them to complete the questionnaires, including a participation number and an internet link to the self-administered online questionnaires. Should the questionnaire identify 
a woman in need of immediate psychological care, that is, find any test score above the thresholds listed above, she will be immediately contacted by phone by the research midwife and encouraged to consult a competent professional (psychologist, psychiatrist, etc) at our hospital who is aware of this research protocol and willing to see these women.

\section{Bias}

To avoid coercion and reduce selection bias, the investigator who will be asking women to participate will not be involved in her direct care. To limit information bias, the patients will complete each questionnaire at the same intervals after delivery (immediate postpartum, 2, 6 and 12 months). The self-administered questionnaires (all validated in French) will be used under the same conditions in which they were validated. Because it is important to take women's anxious personality traits (trait anxiety) before delivery into account, STAI form Y-B will be completed at inclusion.

\section{Study size}

For a power of $90 \%$, with a two-sided alpha of $5 \%$ and two unexposed subjects for each exposed subject, and according to the Australian study by Thompson et al (2011), ${ }^{38}$ which found a depression rate of $10 \%$ in unexposed and $16 \%$ in exposed women at 4 months postpartum, this study requires 1028 women in the unexposed group, with no haemorrhage, and 514 women in exposed group with a haemorrhage. We have chosen this ratio because we think that we are likely to have a substantial rate of loss to follow-up among the unexposed women. A total of 1542 women must therefore be included.

\section{Statistical methods}

Quantitative variables will be expressed by their means $\pm \mathrm{SD}$ or by categorical variables, as appropriate.

The participation and refusal rates will be calculated at each survey point, together with the prevalence of the psychological states studied. Some women will be lost to follow-up at the month 6 and month 12 surveys. These women will be included in the crude descriptive analyses, and population lost to follow-up will be compared with the study population to look for possible differences in the principal characteristics studied. We plan to perform an interim analysis to assess the need to adjust the study calendar. This interim analysis will be performed after half of the planned subjects have been included.

The statistical methods used for the crude analyses will be a descriptive analysis of the sample with a comparison of the characteristics of the exposed and unexposed groups. The $\chi 2$ test (or Fisher's exact test when appropriate) will be used to compare the qualitative variables and Student's t-test (or a Mann-Whitney test) for the quantitative variables.

Prevalence rates and their $95 \%$ CIs will be calculated at 2, 6 and 12 months. The course of the prevalence rates (and their 95\% CI) of depression, anxiety and PTSD at these points will be compared within each of the three survey periods by a matched $\chi 2$ (or McNemar) test.

A multivariate analysis (logistic regression) will be conducted to take into account confounding factors as well as clinically relevant prognostic factors (for example, history of depression, gestational age at birth, mode of delivery, etc) for depression at each survey time point (at 2 months, 6 months and 1 year). The results will be expressed as adjusted ORs. At the end of the study, another multivariate analysis (generalised linear mixed model) will be performed to study the trends in the prevalence of depression over the three study periods. The missing data will be treated as missing and excluded from the analyses.

\section{ETHICS AND DISSEMINATION \\ Protocol amendments}

Any modifications to the protocol must be characterised as substantial or minor. Substantial modifications must be approved de novo by the institutional review board.

\section{Consent}

The women will be informed completely and fairly of the objectives and constraints of the study, of any possible risks, of their right to refuse to participate and of their right to withdraw their consent and end their participation at any time. All of this information will be included on the information and consent form provided to the woman. The investigator shall collect each woman's free and informed consent in writing.

\section{Confidentiality}

In compliance with French regulations, persons with direct access to the data will take all precautions necessary to ensure the confidentiality of the data concerning women participating in this study. Moreover, the storage of data collected for this study must comply with French guidelines issued by the competent agency (CNIL: National Data Protection Authority—http://www.cnil.fr).

The study documents shall be archived on the premises of the PEPRADE research team until the end of their practical utility and then stored in the sponsor's central archives for 15 years. The Clermont-Ferrand University Hospital Centre is the sponsor of this study.

\section{Access to data}

At the conclusion of the research, the data collected about the participants will be anonymised before the statistical analysis. Access to the anonymised data will be limited to the two principal investigators.

\section{Dissemination policy}

The data will be divulged only after the joint accord of the principal investigator and the sponsor. The results will be the subject of scientific communications and publications. The authorship eligibility will follow the Recommendations for the Conduct, Reporting, Editing and 
Publication of Scholarly Work in Medical Journals, 2015 (http://www.icmje.org).

\section{Author affiliations}

${ }^{1}$ Centre Hospitalier Universitaire de Clermont-Ferrand, Clermont-Ferrand, France ${ }^{2}$ UniversitéClermont-Auvergne, CHU Clermont-Ferrand, CNRS,SIGMA Clermont, Institut Pascal, Clermont-Ferrand, France

${ }^{3}$ Psychiatry B, CHU Clermont-Ferrand, Clermont-Ferrand, France

${ }^{4}$ UFR Medecine, EA7280, Univ Clermont 1, Clermont-Ferrand, France

${ }^{5}$ The AUDIPOG Sentinel Network (Association des Utilisateurs de Dossiers informatisés en Pédiatrie, Obstétrique et Gynécologie), RTH Laennec Medical University, Lyon, France

Acknowledgements The authors thank the staff and families for supporting this trial.

Contributors MP and FV designed the study, wrote the study protocol and obtained the funding for the study. MP is the coordinator-investigator and FV the methodologist and supervisor of MP, a PhD student. All the authors (MP, AL, IDC, PML and FV) wrote and approved the final manuscript.

Funding This work was supported by a grant from the Clermont-Ferrand University Hospital (AOI 2015).

\section{Competing interests None declared.}

Ethics approval The PSYCHE study protocol $(\mathrm{V} .9,2016)$ was approved by the institutional review board (IRB) on 14 February 2017 (IRB Sud Est VI: NAU1243), and by the ANSM (National Agency for the Drug and Medical Product Safety) on 03 February 2016 (ID RCB: 2016-A00092-49).

Provenance and peer review Not commissioned; externally peer reviewed.

Open access This is an open access article distributed in accordance with the Creative Commons Attribution Non Commercial (CC BY-NC 4.0) license, which permits others to distribute, remix, adapt, build upon this work non-commercially, and license their derivative works on different terms, provided the original work is properly cited, appropriate credit is given, any changes made indicated, and the use is non-commercial. See: http://creativecommons.org/licenses/by-nc/4.0/.

\section{REFERENCES}

1. Langan R, Goodbred AJ. Identification and management of peripartum depression. Am Fam Physician 2016;93:852-8.

2. Koshchavtsev AG, Mul'tanovskaya VN, Lorer VV. Baby blues syndrome as an adaptation disorder in the early stages of formation of the mother-child system. Neurosci Behav Physiol 2008;38:439-42.

3. Kumar N, Nagaraj AKM, Koudike U, et al. Psychiatric morbidity and correlates in postpartum women in a tertiary care hospital. Indian $J$ Psychol Med 2016:38:309-14.

4. Adewuya AO. The maternity blues in Western Nigerian women: prevalence and risk factors. Am J Obstet Gynecol 2005;193:1522-5.

5. Grussu P, Quatraro RM. Maternity blues in Italian primipara women: symptoms and mood states in the first fifteen days after childbirth. Health Care Women Int 2013;34:556-76.

6. Chung SS, Yoo IY, Joung KH. Post-Partum blues among Korean mothers: a structural equation modelling approach. Int J Ment Health Nurs 2013;22:359-67.

7. Faisal-Cury A, Menezes PR, Tedesco JJA, et al. Maternity "blues": prevalence and risk factors. Span J Psychol 2008;11:593-9.

8. Takahashi Y, Tamakoshi K. Factors associated with early postpartum maternity blues and depression tendency among Japanese mothers with full-term healthy infants. Nagoya J Med Sci 2014;76:129-38.

9. Cox JL, Holden JM, Sagovsky R. Detection of postnatal depression. development of the 10-item Edinburgh postnatal depression scale. Br J Psychiatry 1987;150:782-6.

10. Ferretti F, Franca A, Folin M. [Risk factors associated with postnatal depressive symptomatology: a study conducted in the Southern Area of the Local Health Unit of Modena]. Epidemiol Prev 2013;37:138-44.

11. Petrozzi A, Gagliardi L. Anxious and depressive components of Edinburgh postnatal depression scale in maternal postpartum psychological problems. J Perinat Med 2013;41:343-8.

12. Sword W, Kurtz Landy C, Thabane L, et al. Is mode of delivery associated with postpartum depression at 6 weeks: a prospective cohort study. BJOG Int J Obstet Gynaecol 2011;118:966-77.

13. McCoy SJB, Beal JM, Saunders B, et al. Risk factors for postpartum depression: a retrospective investigation. $J$ Reprod Med 2008;53:166-70.
14. Chaaya M, Campbell OMR, El Kak F, et al. Postpartum depression: prevalence and determinants in Lebanon. Arch Womens Ment Health 2002;5:65-72.

15. Gonidakis F, Rabavilas AD, Varsou E, et al. A 6-month study of postpartum depression and related factors in Athens Greece. Compr Psychiatry 2008;49:275-82.

16. Bahadoran P, Oreizi HR, Safari S. Meta-Analysis of the role of delivery mode in postpartum depression (Iran 1997-2011). J Educ Health Promot 2014;3:118.

17. Upadhyaya S, Sharma A, Raval C. Postpartum psychosis: risk factors identification. N Am J Med Sci 2014;6:35-7.

18. Kapfhammer H-P, Reininghaus EZ, Fitz W, et al. Clinical course of illness in women with early onset puerperal psychosis: a 12-year follow-up study. J Clin Psychiatry 2014;75:1096-104.

19. Ortiz Collado MA, Saez M, Favrod J, et al. Antenatal psychosomatic programming to reduce postpartum depression risk and improve childbirth outcomes: a randomized controlled trial in Spain and France. BMC Pregnancy Childbirth 2014;14:22.

20. Gaillard A, Le Strat $Y$, Mandelbrot L, et al. Predictors of postpartum depression: prospective study of 264 women followed during pregnancy and postpartum. Psychiatry Res 2014;215:341-6.

21. Söderquist J, Wijma B, Thorbert G, et al. Risk factors in pregnancy for post-traumatic stress and depression after childbirth. BJOG Int $J$ Obstet Gynaecol 2009;116:672-80.

22. Czarnocka J, Slade P. Prevalence and predictors of posttraumatic stress symptoms following childbirth. Br J Clin Psychol 2000;39:35-51.

23. Adewuya AO, Ologun YA, Ibigbami OS. Post-Traumatic stress disorder after childbirth in Nigerian women: prevalence and risk factors. BJOG 2006;113:284-8.

24. Zaers S, Waschke M, Ehlert U. Depressive symptoms and symptoms of post-traumatic stress disorder in women after childbirth. $J$ Psychosom Obstet Gynaecol 2008;29:61-71.

25. Olde E, van der Hart O, Kleber R, et al. Posttraumatic stress following childbirth: a review. Clin Psychol Rev 2006;26:1-16.

26. Yildiz PD, Ayers S, Phillips L. The prevalence of posttraumatic stress disorder in pregnancy and after birth: a systematic review and metaanalysis. J Affect Disord 2017;208:634-45.

27. Stuart S, Couser G, Schilder K, et al. Postpartum anxiety and depression: onset and comorbidity in a community sample. J Nerv Ment Dis 1998;186:420-4.

28. Andersson L, Sundström-Poromaa I, Wulff M, et al. Depression and anxiety during pregnancy and six months postpartum: a follow-up study. Acta Obstet Gynecol Scand 2006;85:937-44.

29. Shlomi Polachek I, Huller Harari L, Baum M, et al. Postpartum anxiety in a cohort of women from the general population: risk factors and association with depression during last week of pregnancy, postpartum depression and postpartum PTSD. Isr J Psychiatry Relat Sci 2014;51:128-34.

30. Söderquist J, Wijma K, Wijma B. Traumatic stress in late pregnancy. $J$ Anxiety Disord 2004;18:127-42.

31. Rowlands IJ, Redshaw M. Mode of birth and women's psychological and physical wellbeing in the postnatal period. BMC Pregnancy Childbirth 2012;12:138.

32. Milgrom J, Gemmill AW, Bilszta JL, et al. Antenatal risk factors for postnatal depression: a large prospective study. J Affect Disord 2008;108:147-57.

33. Furuta M, Sandall J, Bick D. A systematic review of the relationship between severe maternal morbidity and post-traumatic stress disorder. BMC Pregnancy Childbirth 2012;12:125.

34. Vendittelli F, Barasinski C, Pereira B, et al. Incidence of immediate postpartum hemorrhages in French maternity units: a prospective observational study (HERA study). BMC Pregnancy Childbirth 2016;16:242.

35. Sentilhes L, Vayssière C, Deneux-Tharaux C, et al. Postpartum hemorrhage: guidelines for clinical practice from the French College of gynaecologists and obstetricians (CNGOF): in collaboration with the French Society of anesthesiology and intensive care (SFAR). Eur J Obstet Gynecol Reprod Biol 2016;198:12-21.

36. Thompson JF, Ford JB, Raynes-Greenow CH, et al. Women's experiences of care and their concerns and needs following a significant primary postpartum hemorrhage. Birth 2011;38:327-35.

37. Sentilhes L, Gromez A, Clavier E, et al. Long-Term psychological impact of severe postpartum hemorrhage. Acta Obstet Gynecol Scand 2011;90:615-20.

38. Thompson JF, Roberts CL, Ellwood DA. Emotional and physical health outcomes after significant primary post-partum haemorrhage (PPH): a multicentre cohort study. Aust N Z J Obstet Gynaecol 2011;51:365-71. 
39. Guedeney N, Fermanian J. Validation study of the French version of the Edinburgh postnatal depression scale (EPDS): new results about use and psychometric properties. Eur Psychiatry 1998;13:83-9.

40. Sheehan DV, Lecrubier Y, Sheehan KH, et al. The Mini-International neuropsychiatric interview (M.I.N.I.): the development and validation of a structured diagnostic psychiatric interview for DSM-IV and ICD10. J Clin Psychiatry 1998:59 Suppl 20:22-33.

41. Spielberger CD, Vagg PR. Psychometric properties of the STAI: a reply to Ramanaiah, Franzen, and Schill. J Pers Assess 1984;48:95-7.

42. Micallef J, McGlangeaud-Freudenthal N, Aurran Y, et al. [Measurement of anxiety state in women: a short-form scale]. Rev Epidemiol Sante Publique 1998;46:383-9.

43. Löwe B, Decker $\mathrm{O}$, Müller $\mathrm{S}$, et al. Validation and standardization of the generalized anxiety disorder screener (GAD-7) in the general population. Med Care 2008;46:266-74.

44. Swinson RP. The GAD-7 scale was accurate for diagnosing generalised anxiety disorder. Evid Based Med 2006;11:184.
45. Micoulaud-Franchi J-A, Lagarde S, Barkate G, et al. Rapid detection of generalized anxiety disorder and major depression in epilepsy: validation of the GAD-7 as a complementary tool to the NDDI-E in a French sample. Epilepsy Behav 2016;57:211-6.

46. Weiss DS, Marmar CR. The Impact of Event Scale-Revised. In: Wilson JP, Keane TM, eds. Assessing Psychological Trauma and PTSD: A Practitioner's Handbook. New York, London: The Guilford Press, 1997: 399-411.

47. Brunet A, St-Hilaire A, Jehel L, et al. Validation of a French version of the impact of event scale-revised. Can J Psychiatry 2003;48:56-61.

48. Beck JG, Grant DM, Read JP, et al. The impact of event scalerevised: psychometric properties in a sample of motor vehicle accident survivors. J Anxiety Disord 2008;22:187-98.

49. Spitzer RL, Kroenke K, Williams JBW, et al. A brief measure for assessing generalized anxiety disorder: the GAD-7. Arch Intern Med 2006;166:1092-7. 